\title{
Predicción de la reincidencia con delincuentes juveniles: un estudio longitudinal
}

\section{Prediction of recidivism in juvenile offenders: a longitudinal study}

Recibido el 11 abril 2016/Publicado el 15 febrero 2017

Enrique López Martín

Comunidad Autónoma de Murcia

Vicente Garrido Genovés ${ }^{1}$

Universidad de Valencia

Juan José López García

Universidad de Murcia

María Jesús López Latorre

Universidad de Valencia

María José Galvis Doménech

Universidad de Valencia

\section{RESUMEN}

El presente trabajo tiene como finalidad determinar la utilidad de una escala en la predicción de la reincidencia de los delincuentes juveniles. Se realizó un estudio longitudinal con una muestra de 258 delincuentes juveniles que se hallaban cumpliendo una medida judicial. El instrumento que evaluó la predicción de la reincidencia fue el Youth Level of Service/Case Management Inventory, cuyo resultado de su adaptación al castellano es el Inventario de Gestión en Intervención para Jóvenes (IGI-J). Los jóvenes

\footnotetext{
${ }^{1}$ La correspondencia debe enviarse a: vicente.garrido@uv.es
} 
delincuentes reincidentes y no reincidentes puntuaron de la forma esperada en los valores obtenidos en la prueba. No obstante, los resultados indican que el IGI-J identifica a todos los sujetos no reincidentes, pero fracasó en la identificación de los reincidentes. Se analiza la posible razón de este resultado así como la contribución de esta prueba en el contexto de la predicción del riesgo en menores y en la elaboración de programas de intervención.

Palabras clave: IGI-J, juicio clínico estructurado, delincuentes juveniles, predicción de la reincidencia, programas de intervención

\begin{abstract}
This paper presents a study oriented to analyze the utility of a scale in the prediction of juvenile offenders' recidivism. It was carried out a longitudinal study in a sample of 258 juvenile offenders that were serving a educative measure imposed by a juvenile court. The selected instrument was the Youth Level of Service/Case Management Inventory, adapted to spanish with the tittle of Inventario de Gestión en Intervención para Jóvenes (IGI-J). The recidivist juvenile offenders showed a higher score than the non-recidivist, but the inventory failed in order to predict with accuracy the subjects that committed a new offence after two years of follow-up. It is analyzed the possible causes of this result, as well as the contribution of the IGI-J in the context of the risk assessment in juvenile justice and in the elaboration of programs of intervention.
\end{abstract}

Key words: IGI-J, structured clinical judgment, juvenile offenders, prediction of recidivism, intervention programs

\title{
1. Introducción
}

La evaluación del riesgo de reincidencia tanto en delincuentes adultos como juveniles ha ido cambiando en los últimos 20 años, viéndose sustituido el término peligrosidad por el de riesgo de violencia (Andrés-Pueyo y Echeburúa, 2010). En el caso de éste último, el objetivo es adaptarse a las exigencias del Sistema de Justicia Juvenil, desarrollando instrumentos de valoración perfeccionados que identifiquen las necesidades criminógenas de los menores infractores (Otto y Douglas, 2010; Welsh, Shmidt, McKinnon, Chattha, y Meyers, 2008). De este modo, se pretende la consolidación de instrumentos de evaluación y programas de intervención que atiendan al interés superior del menor, cumpliendo un papel fundamental respecto a las premisas de reeducación y reinserción social.

Tradicionalmente, la evaluación del riesgo se basaba en la experiencia y juicio del profesional, lo que se conoce como evaluación clínica no estructurada (Andrés-Pueyo y Redondo, 2007). Conceptualmente, esta primera etapa fue superada cuando se diseñaron 
instrumentos que permitían un sistema actuarial de predicción del riesgo (Bengtson y Langström, 2007; Heilbrun, 2009) utilizando para ello los factores estáticos y dinámicos más asociados por la investigación con la continuidad de la conducta delictiva (Andrews y Bonta, 2010). En la actualidad, el método actuarial coexiste con los instrumentos de última generación, que emplean el juicio clínico estructurado (SCJ). La investigación ha mostrado una mayor fiabilidad y validez predictiva del SCJ en comparación con los instrumentos de evaluación no estructurados (Guy, 2008; Olver, Stockdale, y Wormith, 2014; Yang, Wong, y Coid, 2010), dado que combina medidas actuariales y el juicio profesional (Borum, Lodewikjs, Bartel, y Forth, 2010).

Por este motivo, en el presente estudio se emplea el instrumento de "última generación" YLS/CMI en su adaptación al castellano con el objetivo de predecir el riesgo de reincidencia juvenil en una muestra de menores infractores en España utilizando un estudio longitudinal. En España son muy escasos los estudios longitudinales en Criminología, los cuales son imprescindibles para poder validar instrumentos que aspiran a establecer niveles de riesgo sobre los cuales poder posteriormente determinar los objetivos de intervención más apropiados a cada caso (Vincent, Guy, Fusco, y Gershenson, 2012). En este artículo nos vamos a centrar en determinar la capacidad del YLS/CMI (IGI-J en su adaptación española) para predecir la reincidencia futura en delincuentes juveniles.

La escala Level of Service Inventory-Revised (LSI-R; Andrews y Bonta, 1995) es un instrumento actuarial de valoración del riesgo del delincuente adulto basado en los principios de necesidad-riesgo (Andrews, Bonta, y Hoge, 1990; Andrews y Bonta, 2002). Éste recoge las cuatro grandes áreas que mejor predicen la conducta delictiva: historia delictiva, apoyo social para cometer delitos, actitudes u orientación procriminal y las características personales y emocionales más importantes del individuo (Andrews y Bonta, 1994). Desde la primera investigación donde se demostró la validez predictiva de este instrumento, han sido muchos los artículos publicados donde se desarrollaban sus posibilidades de aplicación (Andrews, 1982; Bonta, 1989; Chenane, Brennan, Steiner, y Ellison, 2015; Nguyen, Arbach-Lucioni, y Andrés-Pueyo, 2011; Raynor, Kynch, Roberts, y Merrington, 2000; Ryan, Labrecque, Smith, Lovins, y Latessa, 2014; Singh et al., 2014). La versión para jóvenes de dicho instrumento se corresponde con el Youth Level of Service/Case Management Inventory (YLS/CMI; Hoge y Andrews, 2002), el cual emplea 
el juicio clínico estructurado para evaluar el riesgo general de reincidencia en menores infractores, y se diferencia de su antecesor por la composición de los ítems en función de la edad de los sujetos, otorgando mayor importancia a la familia y a los problemas sociales, y menos al empleo. Está constituido por 42 ítems dicotómicos, organizados en ocho dominios de factores de riesgo: historial delictivo, circunstancias familiares, educación, relación entre iguales, consumo de sustancias tóxicas, ocio/diversión, personalidad y conducta, y actitudes, valores y creencias. El estudio de los factores de riesgo otorga información respecto a la intensidad requerida en la supervisión de los jóvenes, resultando patente la consideración del principio de necesidad en el instrumento YLS/CMI, el cual se asocia con reducciones de la reincidencia (Campbell et al., 2014; Luong y Wormith, 2011; Gutierrez, Wilson, Rugge, y Bonta, 2013; Vincent, PaivaSalisbury, Cook, Guy, y Perrault, 2012). La prueba está diseñada para colaborar en la toma de decisiones que deben realizar los profesionales que se encargan de la ejecución de las medidas. El profesional es el responsable último de establecer el nivel de riesgo, los objetivos y las actividades educativas dirigidas a reducir el riesgo de reincidencia; ésta debe ser revisada cada seis meses (Hoge y Andrews, 2002). La adaptación del mismo al castellano se corresponde con el instrumento IGI-J, acrónimo de Inventario de Gestión e Intervención para Jóvenes (Garrido, López, y Silva, 2004; Garrido, López, Silva, López, y Molina, 2006).

El IGI-J es, actualmente, uno de los instrumentos más utilizados en el Sistema de Justicia Juvenil de España (Arbach-Lucioni y Andrés-Pueyo, en prensa), junto con la adaptación del Structured Assessment for Violence Risk in Youth (SAVRY; Borum, Bartel, y Forth, 2000). El SAVRY es un instrumento que también emplea el juicio profesional estructurado, y que está conformado por 24 ítems agrupados en tres dominios. Éstos son: 10 factores de riesgo históricos, 6 sociales/contextuales y 8 individuales, y cada factor puntúa en una escala del 0 al 3, que se corresponde con un riesgo bajo, medio o elevado. El SAVRY ha sido escogido por el Sistema de Justicia Juvenil de Cataluña como instrumento de referencia (Vallès-Port y Hilterman, 2006). Según el estudio de Childs, Frick, Ryals, Lingonblad, y Villio (2014), la validez del SAVRY se fundamenta en el empleo del juicio clínico estructurado, además de ser considerado uno de los pocos instrumentos que incorporan factores de protección (en seis ítems). 
En determinados casos, se ha destacado la necesidad de valorar no sólo el nivel de riesgo del delincuente juvenil, sino también la presencia de los rasgos de personalidad antisocial y psicopatía, ya que se estima que dichos rasgos cualifican la predicción y el tratamiento que se les debe asignar. Para ello, se han traducido y adaptado para los jóvenes españoles el denominado Antisocial Process Screening Device (APSD; Frick y Hare, 2001; adaptación española por González, Molinuevo, Pardo, y Torrubia, 2003) y la Psychopathy Checklist: Youth Version (PCL: YV; Forth, Kosson, y Hare, 2003; adaptación española de Graña, Garrido, y González, 2011). La PCL: YV se creó a partir de la Escala de Evaluación de Psicopatía de Hare Revisada (Psychopathy ChecklistRevised-PCL-R; Hare, 1991; 2003), y evalúa características psicopáticas en jóvenes de 12 a 18 años. Se compone de 20 ítems que puntúan en una escala del 0 al 3, divididos en cuatro dominios: interpersonal, afectivo, estilo de vida y conducta antisocial. Los datos se extraen de una entrevista y de la información colateral existente sobre el individuo.

Analizando los estudios realizados hasta el momento con el YLS/CMI, se observa que este instrumento es comparado con otras escalas de evaluación del riesgo de violencia, como son la PCL: YV y el SAVRY. Los estudios iniciales con diseño prospectivo entre el YLS/CMI y la PCL: YV compararon la capacidad predictiva de ambos, examinando la reincidencia general y la reincidencia violenta (Catchpole y Gretton, 2003; McKinnon, 2004; Viljoen, McLachlan, y Vincent, 2010). El resultado obtenido en un metaanálisis que combinó 21 muestras fue que tanto para predecir la reincidencia general como la reincidencia violenta, la PCL: YV podía compararse en efectividad con el YLS/CMI. Las puntuaciones de reincidencia general para la PCL: YV y el YLS/CMI fueron de $r_{w}=.27, y .25$, respectivamente, mientras que en la reincidencia violenta, la PCL: YV obtuvo un valor $\mathrm{r}_{\mathrm{w}}=.24$, por $\mathrm{r}_{\mathrm{w}}=.21$ para el YLS/CMI (Edens, Campbell, y Weir, 2006). No obstante, el YLS/CMI evalúa factores dinámicos en siete de las ocho subescalas que contiene, lo que presenta una ventaja sobre la PCL: YV en términos de establecer objetivos de intervención.

Otro estudio posterior de Welsh et al. (2008) comparó tres instrumentos estructurados de evaluación del riesgo en adolescentes (YLS/CMI, SAVRY y la PCL: YV) en cuanto a la validez predictiva e incremental con respecto a la reincidencia general y violenta. Los resultados obtenidos fueron que estas escalas predijeron la reincidencia general y violenta con distintos grados de exactitud, siendo el SAVRY el que mayor 
validez predictiva incremental presentó para la reincidencia general $\left(\mathrm{R}^{2}=.09\right)$ y violenta $\left(\mathrm{R}^{2}=.14\right)$, seguida de la PCL: $\mathrm{YV}\left(\mathrm{R}^{2}=.22 \mathrm{y} \mathrm{R}^{2}=.12\right.$, respectivamente).

Posteriormente, en un metaanálisis realizado por Olver et al. (2009) con 44 muestras que incluían a 8.746 delincuentes juveniles que comparaba el YLS/CMI, la PCL: YV y el SAVRY, se constató que las tres pruebas predecían ligeramente mejor la reincidencia general (valores de $\mathrm{r}_{\mathrm{w}}=.32 ; .28$ y .32 , respectivamente) que la reincidencia violenta (valores $r_{w}=.26, .25$ y .30 , respectivamente). Como se puede observar, los tres instrumentos mostraron una capacidad predictiva muy semejante.

En otra investigación posterior se realizó una comparación de la capacidad predictiva entre el YLS/CMI, el SAVRY y la PCL: YV durante un periodo medio de seguimiento de 10 años (Schmidt, Campbell y Holding, 2011). Cada instrumento predijo la reincidencia general de forma moderada (YLS/CMI: AUC=0.66) (SAVRY: AUC= 0.74; PCL: YV AUC=0.79). Sin embargo, no hubo diferencias significativas en la validez predictiva entre los tipos de reincidencia, y los tres instrumentos fueron mejores en la predicción de la reincidencia en los hombres que en las mujeres (Schmidt, Campbell, y Holding, 2011). De forma similar, investigadores en Singapur trataron de determinar la reincidencia con el YLS/CMI y el SAVRY en una muestra de infractores que pertenecían a bandas juveniles y otros jóvenes que actuaban en solitario (Meng, Daffern, Thomas, y Ying, 2012). Obtuvieron que los jóvenes que sí pertenecían a bandas presentaban un riesgo mayor de reincidencia general (YLS/CMI t(163) $=4.23, \mathrm{p}=0.001, \mathrm{~d}=0.70$ ) y violenta (SAVRY $\mathrm{t}(161)=5.42, \mathrm{p}<0.001, \mathrm{~d}=.91)$ que aquellos que no estaban integrados en bandas. Recientemente, los autores Shepherd, Luebbers, Ogloff, Fullam, y Dolan (2014) apuntaron que tanto el SAVRY como el YLS/CMI y la PCL:YV predicen de manera similar la reincidencia general (SAVRY: AUC $=0.71, \mathrm{p}<0.001$; YLS/CMI: AUC $=$ $.71, \mathrm{p}<.001 ;$ PCL:YV: AUC $=.66, \mathrm{p}<.01)$ y la reincidencia violenta (SAVRY: AUC=.66, $\mathrm{p}<.001 ;$ YLS/CMI: AUC=.66, p<.01; PCL:YV: AUC= .64, p<.01). No obstante, las puntuaciones totales, tanto para el SAVRY como para el YLS/CMI, mostraron mayor precisión predictiva para la reincidencia que la puntuación total de la PCL: YV.

Finalmente, en un estudio realizado en España por Hilterman, Nicholls, y Van Nieuwenhuizen (2014), relativo a los instrumentos SAVRY, YLS/CMI y PCL: YV, participaron 105 menores infractores que estaban cumpliendo una medida judicial de libertad vigilada, con un periodo de seguimiento de un año. El resultado fue que los tres 
instrumentos obtuvieron buenos niveles de validez predictiva al evaluar la probabilidad de reincidencia, no difiriendo la puntuación de riesgo total del SAVRY significativamente del YLS/CMI y la PCL: YV en la evaluación de la reincidencia violenta $(z=0.46 ; p>.05$ y z=0.80; $>.05$, respectivamente), ni tampoco entre la validez predictiva del YLS/CMI y la valoración de la PCL: YV ( $=0.50 ; \mathrm{p}>.05)$. En cuanto a la exactitud predictiva de la evaluación del riesgo de reincidencia en general, hubo diferencias significativas entre las tres herramientas (SAVRY-YLS/CMI: z=0.04; p>.05; SAVRY-PCL: YV: z=0.36; p>.05; YLS/CMI-PCL: YV: z=0.63; p>.05). La puntuación total de la consistencia interna de los instrumentos fue muy elevada, de alpha= .90 (SAVRY y YLS/CMI) y .91 (PCL: YV).

Anteriormente a esta investigación, otros estudios en España ya utilizaron el IGIJ. En la Comunidad Autónoma de Madrid se llevó a cabo una investigación prospectiva de la reincidencia por Graña et al. (2007), cuyo objetivo era evaluar factores de riesgo estáticos y dinámicos que permitieran explicar la conducta delictiva de 208 menores infractores, y con ello, desarrollar objetivos de intervención educativo-terapéuticos. El instrumento mostró importantes indicadores de fiabilidad (alpha de Cronbach .88) y validez predictiva (0.72). Por otro lado, Garrido (2009) realizó un estudio en la Comunidad de Cantabria sobre una muestra de 92 jóvenes que estaban cumpliendo, generalmente, medidas comunitarias impuestas por el juzgado de menores. Se emplearon de forma comparativa los instrumentos IGI-J, la PCL: YV y la escala APSD para evaluar el riesgo de reincidencia. Se obtuvieron valores elevados en cuanto a la fiabilidad de los instrumentos (valores alpha 0.803, 0.759 y 0.787 , respectivamente), y una validez predictiva moderada en los dos primeros instrumentos $(\mathrm{AUC}=0.659$ y 0.699 , y baja en el tercero (0.589). Seguidamente, en la evaluación realizada por los autores Cuervo et al. (2009) en los Juzgados de Menores de Castellón a 210 menores infractores durante un periodo de seguimiento de 6 meses, los análisis estadísticos mostraron diferencias significativas entre los diferentes niveles de predicción del riesgo de reincidencia y la reincidencia real posterior: la mayoría de los reincidentes (90\%) fueron predichos correctamente, mientras que el $70 \%$ de los no reincidentes también fueron identificados correctamente. El IGI-J también mostró una alta consistencia interna (alpha de Cronbach= $.81)$.

En resumen, atendiendo a los estudios previos con el YLS/CMI tanto en culturas occidentales como orientales (Andrews et al., 2012; Meng et al., 2015; Meng, Yu, Lee, y 
Zeng, 2014; Olver, Stockdale, y Wong, 2012; Onifade et al., 2014; Rennie y Dolan, 2010; Schwalbe, 2008; Takahashi, Mori, y Kroner, 2013) puede considerarse que dicho instrumento predice adecuadamente la reincidencia general en comparación con la PCL: YV y el SAVRY, mientras que dicha consideración no siempre se mantiene para la reincidencia violenta. Sin embargo, el YLS/CMI obtiene una consistencia interna muy elevada en la mayor parte de los estudios; el hecho de que además, de una manera única, vincule los factores de riesgo a la elaboración de los objetivos y los programas de intervención, hace que, a nuestro juicio, sea un instrumento importante para la actuación especializada en el ámbito del sistema de justicia juvenil en España.

\section{Método}

En sentido general, nuestro trabajo tenía como objetivo último dotar al sistema de Justicia Juvenil de la Comunidad Autónoma de Murcia de una escala fiable y válida para la predicción de la delincuencia. De modo más específico, la investigación aquí comentada tenía por objeto evaluar la capacidad del IG-J en la predicción prospectiva de la reincidencia de los delincuentes juveniles. Con este fin, se planteó la siguiente doble hipótesis:

a) Los valores de riesgo obtenidos por los delincuentes reincidentes y no reincidentes variarán de manera estadísticamente significativa.

b) El IGI-J será capaz de predecir la reincidencia prospectiva de modo estadísticamente significativo.

\subsection{Participantes}

En este estudio se empleó una muestra de 258 jóvenes infractores que se hallaban cumpliendo una medida judicial impuesta por el Juzgado de Menores de la provincia de Murcia. De éstos, 230 eran varones (89.1\%) y 28 mujeres (10.9\%), con edades comprendidas entre los 14 y 21 años. Los criterios de inclusión fueron: haber cometido un hecho delictivo entre los 14 y los 18 años, encontrarse en proceso de ejecución de una medida judicial tanto no privativa de libertad como de internamiento (régimen cerrado, semiabierto, abierto o terapéutico), y que la medida judicial fuese superior a 50 horas en 
los casos que se impusiera la prestación en beneficio de la comunidad, o de 30 días para el resto de medidas.

\subsection{Instrumentos}

Los instrumentos empleados fueron el YLS/CMI, en su adaptación al castellano: IGI-J (Garrido et al., 2004), y el CRIM (Cuadernillo de Recogida de Información sobre el Menor), realizado por el equipo del Servicio de Ejecución de Medidas Judiciales de Menores de la Comunidad Autónoma de la Región de Murcia. Ambos instrumentos fueron cumplimentados tras la realización de entrevistas en profundidad, vaciado de expediente y de recabar la restante información a través de personas claves (familia, profesores o trabajadores sociales) y otros documentos administrativos (como el expediente único administrativo del joven).

\subsection{Procedimiento}

La recogida de información se realizó adaptándola al proceso metodológico de la ejecución de las medidas judiciales. En este proceso, se realizaron distintas actuaciones para conseguir implantar las pruebas entre los equipos y lograr una aplicación homogénea del instrumento. El primer paso consistió en la presentación y entrenamiento en los instrumentos durante dos días a los profesionales que estaban en los equipos de ejecución, a cargo de uno de los autores de este trabajo. En segundo lugar, se procedió con la valoración interjueces, donde los administradores de los instrumentos fueron supervisados para afianzar el proceso de evaluación y el hecho de compartir los mismos criterios. Para valorar la reincidencia prospectiva, se consideró el hecho de tener una medida judicial impuesta en los dos años posteriores a la evaluación inicial de los sujetos. El criterio de reincidencia fue tener constancia de que el menor había vuelto a ser objeto de una medida judicial dentro de la Comunidad de Murcia.

\subsection{Análisis de datos}

Se llevó a cabo un estudio descriptivo para analizar las características sociodemográficas y demás datos obtenidos con el CRIM. Además, para la comprobación de las hipótesis se 
utilizaron el análisis de varianza y el análisis de regresión logística, así como se empleó el análisis de la curva ROC en el cálculo de la capacidad predictiva del instrumento. Todas estas pruebas se llevaron a cabo con el paquete estadístico SPSS 15.0.

\section{Resultados}

Por lo que respecta a los datos descriptivos, la distribución por edad y sexo fue la siguiente (Figura 1): el 11,2\% de los sujetos tenía 14 ó 15 años (3\% de mujeres y 12\% de varones), el $75 \%$ se situaba entre los 16 y 18 años ( $86 \%$ de mujeres y $73 \%$ de varones), y el $16 \%$ restante presentaba más de 18 años (11\% de mujeres y 17\% de varones). Es importante tener en cuenta que la alta proporción de jóvenes en la categoría de edad más elevada tiene un impacto considerable en el seguimiento que puede realizarse con posterioridad al cumplimiento de la medida, ya que un menor que entre en el sistema de justicia juvenil en esa franja de edad tiene muchas menos posibilidades de reincidir en el marco de la ley juvenil que los chicos que ingresan en el sistema con edades inferiores.

En cuanto a la actividad delictiva (Figura 2), el 55\% de los jóvenes había cometido sólo un delito ( $53.5 \%$ de los varones y $67.9 \%$ de las mujeres), el $23.6 \%$ dos delitos ( $22.6 \%$ de los varones y $32.1 \%$ de las mujeres), el $8.1 \%$ tres delitos y el $13.3 \%$ restante cuatro o más delitos. Ninguna mujer cometió más de dos delitos. Por tanto, el $45 \%$ de los jóvenes eran reincidentes (46.5\% de los varones y $32.1 \%$ de las mujeres). El tipo de delito más prevalente fue el robo con violencia e intimidación (48.1\%), seguido del robo con fuerza (26.77\%), faltas (13.9\%), hurtos (13.6) y robos (11.2\%) (Figura 3). Por otra parte, hurto, robo y faltas fueron los delitos más frecuentes entre los jóvenes reincidentes (no mostrado en tablas). 


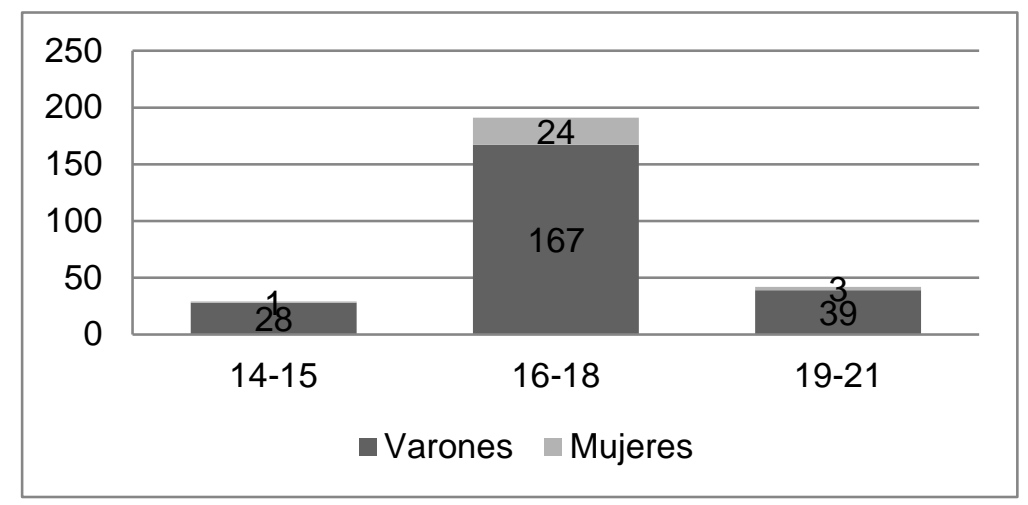

Figura 1: Distribución de la muestra por edad y sexo

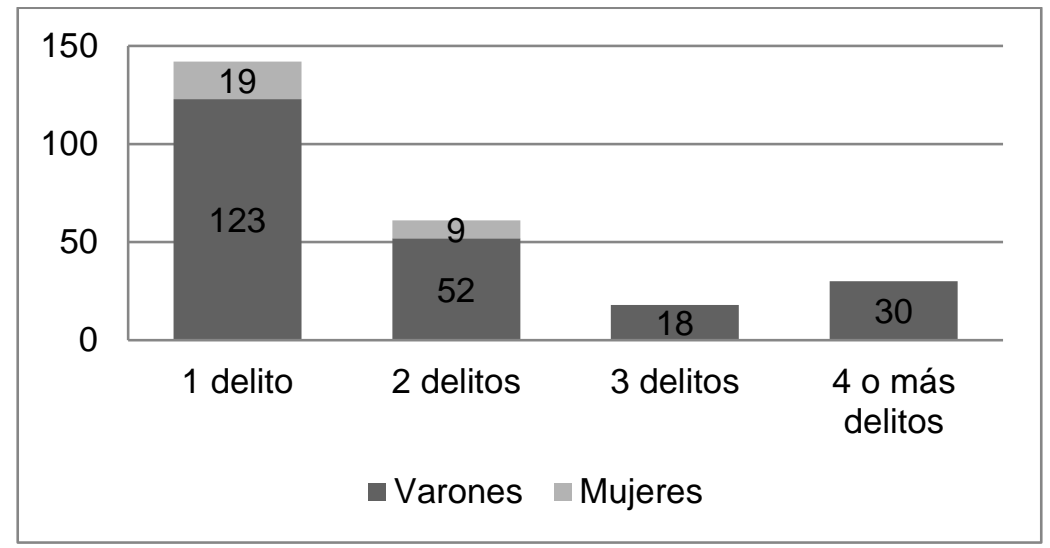

Figura 2: Número de delitos por sexo

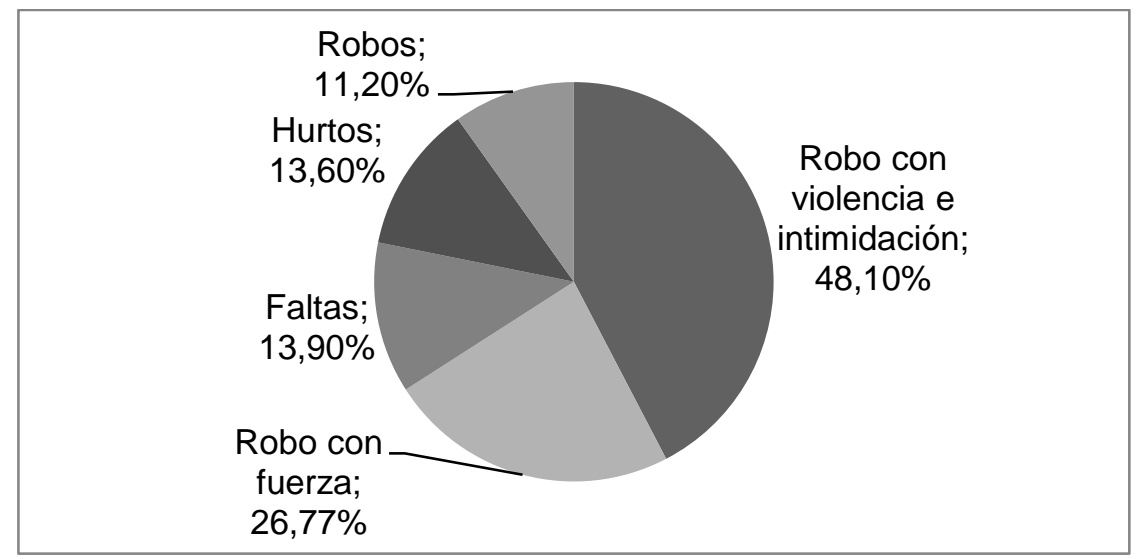

Figura 3: Tipos de delitos y porcentaje de la muestra

Revista Española de Investigación Criminológica

Artículo 6, Número 14 (2016)

www.criminologia.net

ISSN: 1696-9219 
En nuestra investigación, el análisis de fiabilidad del instrumento IGI-J (Tabla 1) arrojó un alpha de Cronbach de .904, utilizando los 42 ítems que lo componen agrupados en 8 subescalas. Los diferentes dominios de riesgo mostraron valores alpha adecuados, entre el rango de 0.61 (ocio/diversión) y 0.77 (actitudes, valores y creencias).

\section{Tabla 1}

Fiabilidad del IGI-J, media y DT del total de la prueba y de cada área por sexo

\begin{tabular}{|l|c|c|c|c|c|c|c|}
\cline { 2 - 8 } & \multirow{2}{*}{$\boldsymbol{\alpha}$ de Cronbach } & \multicolumn{2}{|c|}{ Total N=258 } & \multicolumn{2}{c|}{ Chicos N=230 } & \multicolumn{2}{c|}{ Chicas N=28 } \\
\cline { 3 - 9 } & & Media & DT & Media & DT & Media & DT \\
\hline TOTAL IGI-J (42 ítems) & .90 & 14.37 & 7.97 & 14.74 & 8.04 & 11.32 & 6.69 \\
\hline Total área 1 (5 ítems) & .66 & .91 & 1.20 & .99 & 1.24 & .28 & .46 \\
\hline Total área 2 (6 ítems) & .71 & 2.74 & 1.81 & 2.77 & 1.82 & 2.50 & 1.75 \\
\hline Total área 3 (7 ítems) & .66 & 2.98 & 1.69 & 3.04 & 1.71 & 2.50 & 1.42 \\
\hline Total área 4 (4 ítems) & .63 & 2.08 & 1.18 & 2.11 & 1.20 & 1.85 & 1.04 \\
\hline Total área 5 (5 ítems) & .69 & 1.28 & 1.33 & 1.33 & 1.34 & .89 & 1.19 \\
\hline Total área 6 (3 ítems) & .61 & 1.74 & 1.04 & 1.76 & 1.05 & 1.57 & 1.03 \\
\hline Total área 7 (7 ítems) & .70 & 1.80 & 1.74 & 1.86 & 1.74 & 1.28 & 1.65 \\
\hline Total área 8 (5 ítems) & .77 & .93 & 1.39 & .97 & 1.41 & .53 & 1.23 \\
\hline
\end{tabular}

Nota. Cada área del IGI-J se corresponde con las siguientes subescalas de riesgo: Área 1-Delitos y medidas judiciales pasadas y actuales; Área 2-Pautas educativas; Área 3-Educación formal/empleo; Área 4-Relación con el grupo de iguales; Área 5-Consumo de sustancias; Área 6-Ocio/Diversión; Área 7Personalidad/Conducta; Área 8-Actitudes, Valores y Creencias.

En la misma tabla 1 observamos los valores de la prueba para la muestra total y para las diferentes subescalas o áreas de la prueba. La media del nivel total de riesgo obtenido en la prueba IGI-J de toda la muestra fue de 14,37, y la desviación típica de 7,97. La tabla muestra que, en mujeres $(n=28)$ la media resultó ser 11,32 y la desviación típica 6,69; y en varones, $(n=230)$ estos resultados fueron de 14,74 y 8,04, respectivamente. Las diferencias por sexo fueron estadísticamente significativas $\left(\mathrm{F}_{1.256}=4.66, \mathrm{p}=0.03\right)$.

Por lo que respecta a la reincidencia, el resultado obtenido fue que 207 jóvenes no habían reincidido (un total del $80.2 \%$ de la muestra), mientras que 51 sujetos (19,8\%) sí que lo habían hecho. El nivel de riesgo obtenido en el IGI-J para los segundos fue 17.54 
(DT: 7.43), y para los primeros de 13.59 (DT: 7.92). Esta diferencia fue estadísticamente significativa $\left(\mathrm{F}_{1,241}=9.264 ; p=0.003\right)$.

Ahora bien, hay que tener en cuenta que entre los reincidentes prospectivos, un total de 29, había reincidido con anterioridad a ser evaluados (reincidencia retrospectiva), mientras que los restantes 22 sólo reincidieron en el seguimiento realizado de dos años (lo que da el total de 51 sujetos reincidentes). En ambos subgrupos varían los niveles de riesgo en la dirección esperada. Los reincidentes tanto retrospectivos como prospectivos tienen una media en el IGI-J de 19,21 y una desviación típica de 7,94, mientras que los reincidentes sólo prospectivos tienen una media de 15,36 y una desviación típica de 6.23. En este caso, las diferencias también fueron significativas (Tabla 2).

\section{Tabla 2}

Distribución de la muestra en relación con la reincidencia retrospectiva y prospectiva

\begin{tabular}{|l|c|c|c|}
\hline Resumen & N & Media IGI-J & D.T. \\
\hline No reinciden nunca & 120 & 10.91 & 6.49 \\
\hline Sólo retrospectivamente & 87 & 17.27 & 8.26 \\
\hline Sólo prospectivamente & 22 & 15.36 & 6.23 \\
\hline Tanto Retrospectiva como Prospectivamente & 29 & 19.21 & 7.94 \\
\hline
\end{tabular}

Un análisis inicial consistió en averiguar si la variable "edad” jugaba un papel fundamental en la reincidencia. La edad media de los 207 jóvenes no reincidentes fue 17,85, siendo 16,35 la correspondiente a los reincidentes. La comparación de ambas medias mediante un análisis de varianza confirmó la existencia de diferencias estadísticamente significativas $\left(\mathrm{F}_{1,256}=68.030 ; p=0.000\right)$, lo que permite afirmar que los jóvenes reincidentes tienen una edad significativamente inferior a los jóvenes no reincidentes prospectivamente (Tabla 3) (Se desechó un análisis semejante con la variable sexo debido al número muy reducido de reincidentes mujeres: 9 casos). 


\section{Tabla 3}

Edad según reincidencia prospectiva

\begin{tabular}{|l|l|l|c|}
\hline Reincide Prospectivamente & N & Media & Desviación típica \\
\hline 0: No & 207 & 17.8558 & 1.22842 \\
\hline 1: Sí & 51 & 16.3500 & .87481 \\
\hline Total & 258 & 17.5581 & 1.31128 \\
\hline
\end{tabular}

La correlación de las variables puntuación en el IGI-J y reincidencia prospectiva obtiene un valor bajo: $\mathrm{r}=0.198$, aunque es estadísticamente significativa ( $\mathrm{p}=0.001)$. Por otro lado, cuando se realizó el análisis de regresión logística, se observó que el modelo resultante era significativo ( $\mathrm{p}=0.001$ ), así como el nivel total de riesgo para la escala IGI$\mathrm{J}$ (Tabla 4) para predecir la reincidencia prospectiva $(\mathrm{p}=0.002)$ :

\section{Tabla 4}

Variables en la ecuación de regresión logística

\begin{tabular}{|c|c|c|c|c|c|c|c|c|}
\hline & B & E.T. & Wald & gl & Sig. & Exp(B) & $\begin{array}{c}\text { I.C. 95\% } \\
\text { Inferior }\end{array}$ & $\begin{array}{c}\text { para EXP(B) } \\
\text { Superior }\end{array}$ \\
\hline Nivel de riesgo & 0.060 & 0.019 & 9,903 & 1 & 0.002 & 1,062 & 1,023 & 1,102 \\
\hline Constante & $-2,228$ & 0.348 & 40.917 & 1 & 0.000 & 0.108 & & \\
\hline
\end{tabular}

Por lo que respecta a la capacidad predictiva futura o prospectiva de la prueba, del análisis ROC efectuado desprende que el Área Bajo la Curva es de 0.647 (intervalo de confianza al 95\% 0.51-0.72), el cual resulta estadísticamente significativo $(\mathrm{p}=0.001$; Figura 4); a pesar de que dicho valor demuestra una capacidad predictiva moderada. 


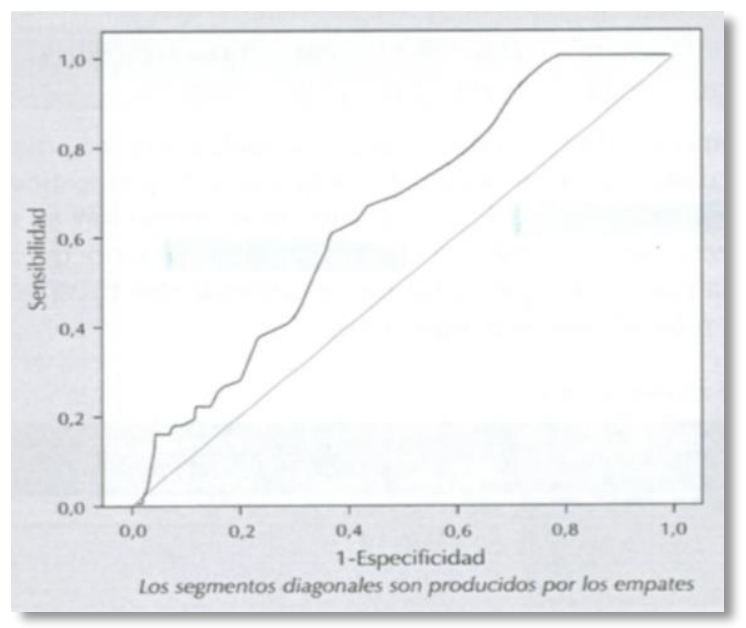

Figura 4: Curva ROC

Sin embargo, como se observa en la tabla de clasificación adjunta (Tabla 5), la predicción no resultó adecuada, ya que todos los sujetos fueron pronosticados como no reincidentes prospectivamente. El alto grado de significación se debió a que se identificaron correctamente a todos los no reincidentes. Uno de los motivos de esta circunstancia puede ser que entre los no reincidentes prospectivamente hubo sujetos que fueron reincidentes retrospectivos, lo que acarreó una reducción del poder predictivo de la prueba en la predicción futura o prospectiva, ya que, como vimos anteriormente, la puntuación en la prueba era superior en los sujetos que habían reincidido tanto en el pasado como en el futuro en comparación con los que sólo habían reincidido en el futuro.

\section{Tabla 5}

Tabla de clasificación en la predicción futura o prospectiva

\begin{tabular}{|c|c|c|c|}
\hline Observado & No reincide pros & Pronosticado Reincide pros & No reincide pros \\
\hline $\begin{array}{c}\text { No reincide } \\
\text { prospectivamente }\end{array}$ & 203 & 0 & 100.0 \\
\hline Reincide pros & 55 & 0 & 0.0 \\
\hline Porcentaje total & & & 78,7 \\
\hline
\end{tabular}

\section{Discusión}


En las últimas décadas, las líneas de investigación seguidas por profesionales e investigadores en el campo de la evaluación del riesgo de violencia han experimentado grandes avances (Andrés-Pueyo y Echeburúa, 2010). En España concretamente, esto se debe a las exigencias del Sistema de Justicia, el cual requería el desarrollo y una utilización consolidada de instrumentos de última generación que realizaran una evaluación holística del riesgo de reincidencia del infractor. Este hecho permite la implementación de programas de intervención que, en el caso de los menores, están orientados a velar por el interés superior de los mismos. En este sentido, la predicción de la reincidencia en población juvenil no ha sido tan ampliamente analizada como en adultos (Andrés-Pueyo y Redondo, 2007; Ballesteros, Graña, y Andreu, 2006), por lo que se requiere una investigación en profundidad que asiente los instrumentos de evaluación más adecuados (Andrés-Pueyo y Echeburúa, 2010). Sin embargo, los hallazgos de diversos metaanálisis ya han respaldado el uso de nuevos constructos que analizan la reincidencia en sujetos jóvenes de forma satisfactoria, como son los instrumentos SAVRY, YLS/CMI y PCL: YV (Catchpole y Gretton, 2003; Edens et al., 2006; McKinnon, 2004; Viljoen et al., 2010).

La presente investigación se planteó como objetivo general "dotar al sistema de Justicia Juvenil de la Comunidad Autónoma de Murcia de una escala fiable y válida para la predicción de la delincuencia" con vistas a que los profesionales encargados de la predicción del riesgo de violencia pudieran trabajar de forma homogénea con un instrumento adecuado a su propósito. El instrumento escogido fue el IGI-J, dado que los estudios realizados hasta la fecha respaldan su adecuación como un buen instrumento de predicción de la reincidencia en jóvenes infractores. En primer lugar, fue necesario corroborar la fiabilidad de dicho instrumento respecto a la predicción prospectiva de la reincidencia en la muestra estudiada. En diversos estudios comparativos en relación a otros instrumentos, las puntuaciones obtenidas oscilan en una horquilla entre 0.56 (Schmidt, Hoge y Gomes, 2005) y 0.78 (Catchpole y Gretton, 2003); mientras que en la presente investigación obtuvimos un alpha de Cronbach de 0.90., superior al de otros trabajos (Onifade et al., 2008; Schmidt, Hoge, y Gomes, 2005). Asimismo, del análisis ROC efectuado se desprende que el Área Bajo la Curva es de .647, el cual resulta también estadísticamente significativo $(\mathrm{p}=0.001)$, hecho que permite atribuir a la prueba una capacidad predictiva moderada. 
De modo más específico, en la investigación presente se planteó una doble hipótesis: los valores de riesgo obtenidos por los delincuentes reincidentes y no reincidentes variarían de manera estadísticamente significativa; y que el IGI-J sería capaz de predecir la reincidencia prospectiva de modo estadísticamente significativo. Respecto a la hipótesis primera, en ambos subgrupos variaron los niveles de riesgo en la dirección esperada. Se corroboró que los reincidentes tanto prospectivos como retrospectivos tenían una media de nivel de riesgo superior $(19,21)$ a los sólo reincidentes prospectivos $(15,36)$. De modo contrario, en la segunda hipótesis la predicción no resultó tan adecuada, ya que se pronosticaron correctamente a todos los no reincidentes prospectivos (203 sujetos), pero fracasó a la hora de predecir los reincidentes prospectivos (55 sujetos).

Con miras hacia el futuro, cabe destacar las limitaciones de la presente investigación con objeto de poder subsanarlas en posteriores trabajos. La primera fue la ausencia de un número suficiente de chicas, lo que dificulta la generalización de los resultados a las delincuentes femeninas. La segunda es la dificultad en realizar un seguimiento de la reincidencia más allá del Sistema de Justicia Juvenil operativo en la Comunidad de Murcia. Aunque pensamos que la mayoría de estos jóvenes cometerá sus delitos en esta región, el hecho de que no pudiéramos obtener datos de las fuerzas policiales y de las instituciones penitenciarias, sin duda supone una merma en el conocimiento real de los reincidentes, lo que puede haber influido en los resultados obtenidos. El número de jóvenes que estaban próximos a la mayoría de edad cuando estaban cumpliendo la medida ha impedido que su posible reincidencia fuera seguida fuera del sistema de justicia juvenil. Debe de considerarse como una limitación en nuestra investigación el hecho de que no se tomaron en consideración los posibles casos de reincidencia en los que el juez pudiera haber tomado la decisión de no procesar al joven por la poca relevancia del delito, o bien porque se hubiera tomado la vía de una decisión extrajudicial.

Por otro lado, y como los datos han indicado, debemos trabajar para intentar mejorar la capacidad predictiva del IGI-J, de modo que disminuyan los falsos negativos obtenidos y se equipare su capacidad predictiva prospectivamente en términos de válidos positivos con instrumentos similares empleados en el contexto de la predicción de la reincidencia con menores infractores (Andrews et al., 2012; Hilterman et al., 2014; Meng et al., 2015; Olver et al., 2012). Sin duda, hemos de considerar decepcionante el 
desempeño del IGI-J en la detección de los reincidentes prospectivos, ya que este es el criterio básico de éxito de un instrumento de predicción del riesgo. Estos datos deben de ponernos en alerta y alentar nuevos estudios donde comprobar si tales resultados han sido excepcionales o responden a determinados elementos del instrumento que hay que revisar y corregir.

No obstante, los resultados obtenidos en la investigación pueden considerarse interesantes debido a la naturaleza del presente instrumento de intervención estructurado. Resulta determinante la incorporación de los principios del riesgo e intervención con los delincuentes juveniles, dado que atiende a una estructura metodológica que permite establecer una lista de factores de riesgo y protección, señalar un nivel de riesgo y un pronóstico de reincidencia, concretar unos objetivos de intervención revisables de acuerdo con los logros de la intervención efectuada, y finalmente adecuar la intensidad de intervención a las exigencias que presentan los jóvenes y su contexto. El hecho de que anteriores trabajos hayan mostrado un nivel de predicción de la reincidencia prospectiva más adecuado que el logrado por nosotros, debería ser un acicate para seguir investigando con este instrumento y ver en qué medida pueden mejorarse nuestros resultados.

\section{Referencias}

Andrés-Pueyo, A., \& Echeburúa, E. (2010). Valoración del riesgo de reincidencia: instrumentos disponibles e indicaciones de aplicación. Psicothema, 22(3), 403409.

Andrés-Pueyo, A., \& Redondo, S. (2007). Violence prediction. Papeles del Psicólogo, 29(1), 107-122.

Andrews, D. A. (1982). The Level of Supervision Inventory (LSI): The first follow-up. Toronto: Ontario Ministery of Correctional Services.

Andrews, D. A., \& Bonta, J. (1994). The psychology of criminal conduct (1st ed.). Cincinnati, $\mathrm{OH}$ : Anderson.

Andrews, D. A., \& Bonta, J. (1995). LSI-R: The Level of Service Inventory-Revised. Toronto: Multi-Health Systems.

Andrews, D. A., \& Bonta, J. (2002). The psychology of criminal conduct (3rd ed.). Cincinnati, $\mathrm{OH}$ : Anderson.

Andrews, D. A., \& Bonta, J. (2010). The psychology of criminal conduct (5th ed.). Cincinnati, $\mathrm{OH}$ : Anderson.

Andrews, D. A., Bonta, J., \& Hoge, R. (1990). Classification for effective rehabilitation: Rediscovering psychology. Criminal Justice and Behavior, 17(1), 19-52.

Andrews, D. A., Guzzo, L., Raynor, P., Rowe, R. C., Rettinger, J., Brews, A., \& Wormith, J. S. (2012). Are the major risk/need factors predictive of both female and male 
reoffending? A test with the eight domains of the Level of Service/Case Management Inventory. International Journal of Offender Therapy and Comparative Criminology, 56(1), 113-133. doi: 10.1177/0306624X10395716

Arbach-Lucioni, K., \& Andrés-Pueyo, A. (en prensa). Violence Risk Assessment Practices in Spain.

Ballesteros, A., Graña, J., \& Andreu, J. (2006). Actuarial violence risk assessment in correctional institutions. Psicopatología Clínica, Legal y Forense, 6(1), 103-117.

Bengtson, S., \& Langström, N. (2007). Unguided clinical and actuarial assessment of reoffending risk: A direct comparison with sex offenders in Denmark. Sexual Abuse: A journal of Research and Treatment, 19(2), 135-153.

Bonta, J. (1989). Native Inmates: institutional response, risk, and needs. Canadian Journal of Criminology, 31(1), 49-62.

Borum, R., Bartel, P., \& Forth, A. (2000). Manual for the Structured Assessment of Violence Risk in Youth. Tampa: University of South Florida.

Borum, R., Lodewikjs, H., Bartel, P., \& Forth, A. (2010). Structured Assessment of Violence Risk in Youth (SAVRY). In R. K. Otto y Douglas (Eds.), Handbook of violence risk assessment (pp.63-80). New York, NY: Routledge.

Campbell, C., Onifade, E., Barnes, A., Peterson, J., Anderson, V., Davidson, W., \& Gordon, D. (2014). Screening Offenders: The Exploration of a Youth Level of Service/Case Management Inventory: (YLS/CMI) Brief Screener. Journal of Offender Rehabilitation, 53(1), 19-34. doi: 10.1080/10509674.2013.861314

Catchpole, R., \& Gretton, H. (2003). The predictive validity of risk assessment with violent young offenders: A 1-year examinaron of criminal outcome. Criminal Justice and Behavior, 30(6), 688-708. doi: 10.1177/0093854803256455

Chenane, J., Brennan, P., Steiner, B., \& Ellison, J. (2015). Racial and ethnic differences in the predictive validity of the Level of Service Inventory-Revised among prison inmates. Criminal Justice and Behavior, 42(3), 286-303. doi: 10.1177/0093854814548195

Childs, K., Frick, P., Ryals, J., Lingonblad, A., \& Villio, M. (2014). A comparation of empirically based and Structured Professional Judgement Estimation of Risk Using the Structured Assessment of Violence Risk in Youth. Youth violence and juvenile justice, 12(1), 40-57. doi: 10.1177/1541204013480368

Cuervo, K., Andrés, C., Gorriz, A., Villanueva, L., Carrión, C., \& Busquets, P. (2009). Predicción de la reincidencia delictiva en menores infractores. International Journal of Developmental and Educational Psychology, (2), 529-538.

Edens, J., Campbell, J., \& Weir, J. (2006). Youth psychopathy and criminal recidivism: a meta-analysis of the psychopathy checklist measures. Law and Human Behavior, 31(1), 53-75. doi: 10.1007/s10979-006-9019-y

Forth, A., Kosson, D., \& Hare, R. (2003). The Psychopathy Checklist: Youth version manual. Toronto: Multi-Health Systems.

Frick, P., \& Hare, R. (2001). The Antisocial Process Screening Device - Technical manual. Toronto: Multi-Health Systems.

Garrido, V. (2009). La predicción y la intervención con los menores infractores: Un estudio en Cantabria. (Estadísticas, instrumentos, protocolos y evaluación). Gobierno de Cantabria, Consejería de Empleo y Bienestar Social. Cantabria: Dirección General de Políticas Sociales.

Garrido, V., López, E., \& Silva, T. (2004). Translation into Spanish of the Youth Level of Service/Case Management Inventory. Valencia: Tirant lo Blanch. 
Garrido, V., López, E., Silva, T., López, M., \& Molina, P. (2006). El modelo de la competencia social de la Ley de Menores. Cómo prevenir y evaluar la intervención educativa. Valencia: Tirant lo Blanch.

González, L., Molinuevo, B., Pardo, Y., \& Torrubia, R. (2003). Authorized Spanish adaptación of the PCL: YV. Barcelona: Universitat Autònoma de Barcelona.

Graña, J., Garrido, V., \& González, L. (2007). The assessment of criminal characteristics of juvenil offenders from the Community of Madrid and its influence in the treatment planning. Psicopatología Clínica, Legal y Forense, 7(1), 7-18.

Graña, J., Garrido, V., \& González, L. (2011). Criminal reoffending in juvenile offenders from the Community of Madrid: assessment, criminal characteristics and prediction models. Madrid: Agencia para la reeducación y reinserción del menor infractor.

Gutierrez, L., Wilson, H., Rugge, T., \& Bonta, J. (2013). The prediction of recidivism with Aboriginal offenders: A theoretically informed meta-analysis. Canadian Journal of Criminology and Criminal Justice, 55(1), 55-99.

Guy, L. (2008). Performance indicators of the structured professional judgement approach for assessing risk for violence to others: A meta-analytic survey. Tesis doctoral no publicada. Simon Fraser University, British Columbia, Canada.

Hare, R. (1991). The Hare Psychopathy Checklist Revised. Toronto: Multi-Health Systems.

Hare, R. (2003). Hare Psychopathy Checklist-Revised manual (2nd ed.). Toronto: MultiHealth Systems.

Heilbrun, K. (2009). Evaluation for risk in violence in adults. New York: Oxford University Press.

Hilterman, E., Nicholls, T., \& Van Nieuwenhuizen, C. (2014). Predictive validity of risk assessments in juvenil offenders: comparing the SAVRY, PCL: YV and YLS/CMI with unestructured clinical assessments. Assessment, 21(3), 324-339. doi: $10.1177 / 1073191113498113$

Hoge, R., \& Andrews, D. A. (2002). Youth Level of Service/Case Management Inventory (YLS/CMI). Toronto: Multi-Health Systems.

Luong, D., \& Wormith, J. (2011). Applying risk/need assessment to probation practice and its impact on the recidivism of young offenders. Criminal Justice and Behavior, 38(12), 1177-1199. doi: 10.1177/0093854811421596

Meng, C., Daffern, M., Thomas, S., \& Ying, L. (2012). Violence risk and gang affiliation in youth offenders: a recidivism study. Psychology, Crime \& Law, 18(3), 299315. doi: 10.1080/1068316X.2010.481626

Meng, C., Yu, H., Lee, Y., \& Zeng, G. (2014). The utility of the YLS/CMI-SV for assessing youth offenders in Singapore. Criminal Justice and Behavior, 41(12), 1437-1457. doi: 10.1177/0093854814537626

Meng, C., Lee, Y., Zeng, G., Yim, G., Yeh, C., Ang, Y., Chin, S., \& Ruby, K. (2015). Assessing Youth Offenders in a Non-Western Context: The predictive Validity of the YLS/CMI Ratings. Psychological Assessment, 27(3), 1013-1021. doi: $10.1037 / \mathrm{a} 0038670$

McKinnon, L. (2004). Predicting risk of violence in a young offender population: The predictive validity of the PCL: $Y V$ and the YLS/CMI. Unpublished master's thesis. Lakehead University, Canada. 
Nguyen, T., Arbach-Lucioni, K., \& Andrés-Pueyo, A. (2011). Factores de riesgo de la reincidencia violenta en población penitenciaria. Revista de Derecho Penal y Criminología, 3(6), 273-294.

Olver, M., Stockdale, K., \& Wong, S. (2012). Short and long-term prediction of recidivism using the YLS/CMI in a sample of serious young offenders. Law and Human Behavior, 36(4), 331-344. doi: 10.1037/h0093927

Olver, M., Stockdale, K., \& Wormith, J. (2009). Risk assessment with young offenders: A meta-analysis of three assessment measures. Criminal Justice and Behavior, 36(4), 329-353. doi: 10.1177/0093854809331457

Olver, M., Stockdale, K., \& Wormith, J. (2014). Thirty years of research on the Level of Service Scales: A meta-Analytic Examination of predictive accuracy and sources of variability. Psychological Assessment, 26(1), 156-176. doi: 10.1037/a0035080

Onifade, E., Davidson, W., Campbell, C., Turke, G., Malinowski, J., \& Turner, K. (2008). Predicting recidivism in probationers with the Youth Level Service/Case Management Inventory (YLS/CMI). Criminal Justice and Behavior, 35(4), 474483. doi: $10.1177 / 0093854807313427$

Onifade, E., Barnes, A., Campbell, C., Anderson, V., Petersen, J., \& Davidson, W. (2014). Juvenile offenders and experiences of neglect: The validity of the YLS/CMI with dual-status youth. Children and Youth Services Review, 46, 112-119. doi: 10.1016/j.childyouth.2014.08.004

Otto, R., \& Douglas, K. (Eds.). (2010). Handbook of violence risk assessment. New York: Taylor \& Francis Group.

Raynor, P., Kynch, J., Roberts, C., \& Merrington, S. (2000). Risk and need assessment in probation services: An evaluation. Londres: Research, Devolopment and Statistics Directorate, Home Office.

Rennie, C., \& Dolan, M. (2010). Predictive validity of the Youth Level of Service/Case Management Inventory in custody sample in England. Journal of Forensic Psychiatry y Psychology, 21(3), 407-425. doi: 10.1080/14789940903452311

Ryan, M., Labrecque, M., Smith, P., Lovins, B., \& Latessa, E. (2014). The Importance of reassessment: How changes in the LSI-R risk score can Improve the prediction of recidivism. Journal of Offender Rehabilitation, 53(2), 116-128. doi: 10.1080/10509674.2013.868389

Schmidt, F., Hoge, R., \& Gomes, L. (2005). Reliability and validity analysis of the Youth Level of Service/Case Management Inventory. Criminal Justice and Behabior, 32(3), 329-344. doi: 10.1177/0093854804274373

Schmidt, F., Campbell, M., \& Holding, C. (2011). Comparative analyses of the YLS/CMI, SAVRY and PCL: YV in adolescent offenders: a 10-year follow-up into adulthood. Youth Violence and Juvenile Justice, 9(1), 23-42. doi: $10.1177 / 1541204010371793$

Schwalbe, C. (2008). A meta-analysis of juvenile justice risk assessment instruments: Predictive validity by gender. Criminal Justice and Behavior, 35(11), 1367-1381. doi: $10.1177 / 0093854808324377$

Shepherd, S., Luebbers, S., Ogloff, J., Fullam, R., \& Dolan, M. (2014). The predictive validity of risk assessment approaches for young australian offenders. Psychiatry, Psychology and Law, 21(5), 801-817. doi: 10.1080/13218719.2014.904262

Singh, J., Desmarais, S., Hurducas, C., Arbach-Lucioni, K., Condemarin, C., Dean, K., ... Otto, K. (2014). International Perspectives on the Practical Application of Violence Risk Assessment: A Global Survey of 44 Countries. International 
Journal of Forensic Mental Health, 13(3), 193-206. doi: 10.1080/14999013.2014.922141

Takahashi, M., Mori, T., \& Kroner, D. (2013). A cross-validation of the Youth Level of Service/Case Management Inventory (YLS/CMI) Among Japanese Juvenile Offenders. Law and Human Behavior, 37(6), 389-400. doi: 10.1037/lhb0000029

Vallès-Port, L., \& Hilterman, E. (2006). SAVRY. Manual of the Spanish version of the Structured Assessment of Violence Risk in Youth. Barcelona, Spain: Centre of Legal Studies and Specialized Training.

Viljoen, J., McLachlan, K., \& Vincent, G. (2010). Assessing violence risk and psychopathy in juvenil and adult offenders: A survey of clinical practices. Assessment, 17(3), 377-395. doi: 10.1177/1073191109359587

Vincent, G., Guy, L., Fusco, S., \& Gershenson, B. (2012). Field reliability of the SAVRY with juvenile probation offenders: Implicaciones for training. Law and Human Behavior, 36(3), 225-236. doi: 10.1037/h0093974

Vincent, G., Paiva-Salisbury, M., Cook, N., Guy, L., \& Perrault, R. (2012). Impact of risk/needs assessment on juvenile probation officers' decision making: Importance of Implementation. Psychology, Public Policy, and Law, 18(4), 549576. doi: 10.1037/a0027186

Welsh, J., Schmidt, F., McKinnon, L., Chattha, H., \& Meyers, J. (2008). A comparative study of adolescent risk assessment instruments' predictive and incremental validity. Assessment, 15(1), 104-115. doi: 10.1177/1073191107307966

Yang, M., Wong, S., \& Coid, J. (2010). The efficacy of violence prediction: A metaanalytic comparison of nine risk assessment instruments. Psychological Bulletin, 136(5), 740-767. doi: 10.1037/a002047

Enrique López Martín es doctor en Psicología por la Universidad de Valencia. Jefe de la Sección de Internamiento del Servicio de Ejecución de Medidas de Justicia Juvenil (CAM).

Vicente Garrido Genovés es Profesor Titular de la Universidad de Valencia. Facultad de Filosofía. Adscrito al Instituto de Creatividad de la Universidad de Valencia.

Juan José López García es profesor titular de la Universidad de Murcia. Facultad de Psicología, Departamento de Psicología Básica y Metodología.

María Jesús López Latorre es profesora titular de la Universidad de Valencia. Facultad de Psicología, Departamento de Psicología Básica.

María José Galvis Doménech es graduada en Criminología y disfruta de una ayuda FPU del Ministerio de Educación y Cultura. Adscrita al Instituto de Creatividad de la Universidad de Valencia. 